\title{
HERMITE-HADAMARD-FEJÉR TYPE INEQUALITIES FOR CONFORMABLE FRACTIONAL INTEGRALS
}

\author{
ERHAN SET AND İLKER MUMCU
}

Received 05 October, 2017

\begin{abstract}
In this work, firstly we have established Hermite-Hadamard-Fejér inequality for conformable fractional integrals. Secondly, we give a new lemma and obtain Hermite-HadamardFejér type integral inequalities for conformable fractional integrals by using this lemma.
\end{abstract}

2010 Mathematics Subject Classification: 26A33; 26D10; 26D15; 33B15

Keywords: Hermite-Hadamard-Fejér inequality, Riemann-Liouville fractional integral, conformable fractional integral

\section{INTRODUCTION}

A function $f: I \subseteq \mathbb{R} \rightarrow \mathbb{R}$ is said to be convex if the inequality

$$
f(\lambda u+(1-\lambda) v) \leq \lambda f(u)+(1-\lambda) f(v)
$$

holds for all $u, v \in I$ and $\lambda \in[0,1]$.

Let $f: I \subseteq \mathbb{R} \rightarrow \mathbb{R}$ be a convex function and $a, b \in I$ with $a<b$, then

$$
f\left(\frac{a+b}{2}\right) \leq \frac{1}{b-a} \int_{a}^{b} f(x) d x \leq \frac{f(a)+f(b)}{2}
$$

is known as the Hermite-Hadamard inequality.

Fejér gave a generalization of the inequalities of (1.1) as the following: If $f$ : $[a, b] \rightarrow \mathbb{R}$ is a convex function, $g:[a, b] \rightarrow \mathbb{R}$ is non-negative, integrable and symmetric to $(a+b) / 2$, then

$$
f\left(\frac{a+b}{2}\right) \int_{a}^{b} g(x) d x \leq \frac{1}{b-a} \int_{a}^{b} f(x) g(x) d x \leq \frac{f(a)+f(b)}{2} \int_{a}^{b} g(x) d x
$$

Definition 1. Let $f \in L_{1}[a, b]$. The Riemann-Liouville integrals $J_{a+}^{\mu} f$ and $J_{b-}^{\mu} f$ of order $\mu>0$ are defined by

$$
J_{a+}^{\mu} f(x)=\frac{1}{\Gamma(\mu)} \int_{a}^{x}(x-t)^{\mu-1} f(t) d t, \quad x>a
$$


and

$$
J_{b-}^{\mu} f(x)=\frac{1}{\Gamma(\mu)} \int_{x}^{b}(t-x)^{\mu-1} f(t) d t, \quad x<b
$$

respectively where $\Gamma(\mu)=\int_{0}^{\infty} e^{-t} u^{\mu-1} d u$. Here $J_{a+}^{0} f(x)=J_{b-}^{0} f(x)=f(x)$.

In the case of $\mu=1$, the fractional integral reduces to the classical integral. We define the Beta function [4, p18]:

$$
B(a, b)=\frac{\Gamma(a) \Gamma(b)}{\Gamma(a+b)}=\int_{0}^{1} t^{a-1}(1-t)^{b-1} d t, \quad a, b>0,
$$

where $\Gamma(\alpha)=\int_{0}^{\infty} e^{-t} u^{\alpha-1} d u$ is the Gamma function.

The incomplete Beta function is defined by

$$
B_{x}(a, b)=\int_{0}^{x} t^{a-1}(1-t)^{b-1} d t, \quad a, b>0,0 \leq x \leq 1
$$

Incomplete Beta function satisfies the following identity

$$
B_{t}(a, b)+B_{1-t}(b, a)=B(a, b)
$$

We use in this paper the Newton-Leibniz formula

$$
\frac{d}{d t}\left(\int_{a(t)}^{b(t)} f(x, t) d x\right)=\int_{a(t)}^{b(t)} \frac{\partial f}{\partial t} d x+f(b(t), t) b^{\prime}(t)+f(a(t), t) a^{\prime}(t)
$$

where $f(x, t)$ be a function such that the partial derivative of $\mathrm{f}$ with respect to $\mathrm{t}$ exists, and is continuous.

In spite of its valuable contributions to mathematical analysis, the RiemannLiouville fractional integrals have deficiencies. For example the solution of the differential equation that is given as

$$
y^{\left(\frac{1}{2}\right)}+y=x^{\left(\frac{1}{2}\right)}+\frac{2}{\Gamma(2.5)} x^{\left(\frac{3}{2}\right)}, y(0)=0
$$

where $y^{\left(\frac{1}{2}\right)}$ is the fractional derivative of $y$ of order $\frac{1}{2}$.

The solution of the above differential equation have caused to imagine on a new and simple representation of the definition of fractional derivative. In [3], Khalil et al. gave a new definition that is called "Conformable fractional derivative". They not only proved further properties of this definitions but also gave the differences with the other fractional derivatives. Besides, another considerable study have presented by Abdeljawad to discuss the basic concepts of fractional calculus. In [1], Abdeljawad gave the following definitions of right-left fractional integrals:

Definition 2. Let $\alpha \in(n, n+1], n=0,1,2, \ldots$ and set $\beta=\alpha-n$ then the left conformable fractional integral starting at $a$ if order $\alpha$ is defined by

$$
\left(I_{\alpha}^{a} f\right)(t)=\frac{1}{n !} \int_{a}^{t}(t-x)^{n}(x-a)^{\beta-1} f(x) d x
$$


Analogously, the right conformable fractional integral is defined by

$$
\left({ }^{b} I_{\alpha} f\right)(t)=\frac{1}{n !} \int_{t}^{b}(x-t)^{n}(b-x)^{\beta-1} f(x) d x .
$$

Notice that if $\alpha=n+1$ then $\beta=\alpha-n=n+1-n=1$ and hence $\left(I_{\alpha}^{a} f\right)(t)=$ $\left(J_{n+1}^{a} f\right)(t)$.

The main purpose of this paper is to establish Hermite-Hadamard-Fejér inequalities for convex functions via conformable fractional integral. We also obtain HermiteHadamard type inequalities of these classes of functions.

\section{HeRmite-HADAMARD-FEJÉR INEQUALITIES FOR CONFORMABLE FRACTIONAL INEQUALITIES}

Throughout this section, let $\|g\|_{\infty}=\sup _{t \in[a, b]}|g(x)|$, for the continuous function $g:[a, b] \rightarrow \mathbb{R}$.

Lemma 1. If $g:[a, b] \rightarrow \mathbb{R}$ is integrable and symmetric to $(a+b) / 2$ with $a<b$, then

$$
I_{\alpha}^{a} g(b)={ }^{b} I_{\alpha} g(a)=\frac{1}{2}\left[I_{\alpha}^{a} g(b)+{ }^{b} I_{\alpha} g(a)\right]
$$

with $\alpha \in(n, n+1], n \in \mathbb{N}$.

Proof. Since $g$ is symmetric to $(a+b) / 2$, we have $g(a+b-x)=g(x)$, for all $g(x)$, for all $x \in[a, b]$. Hence, in the following integral setting $x=a+b-t$ and $d x=-d t$ gives

$$
\begin{aligned}
I_{\alpha}^{a} g(b) & =\frac{1}{n !} \int_{a}^{b}(b-x)^{n}(x-a)^{\alpha-n-1} g(x) d x \\
& =\frac{1}{n !} \int_{a}^{b}(t-a)^{n}(b-t)^{\alpha-n-1} g(a+b-t) d t \\
& =\frac{1}{n !} \int_{a}^{b}(t-a)^{n}(b-t)^{\alpha-n-1} g(t) d t \\
& ={ }^{b} I_{\alpha} g(a) .
\end{aligned}
$$

This completes the proof.

Theorem 1. Let $f:[a, b] \rightarrow \mathbb{R}$ be convex function with $a<b$ and $f \in L[a, b]$. If $g:[a, b] \rightarrow \mathbb{R}$ is non-negative, integrable and symmetric to $(a+b) / 2$, then the following inequalities holds for conformable fractional integrals

$$
\begin{aligned}
f\left(\frac{a+b}{2}\right)\left[I_{\alpha}^{a} g(b)+{ }^{b} I_{\alpha} g(a)\right] & \leq\left[I_{\alpha}^{a}(f g)(b)+{ }^{b} I_{\alpha}(f g)(a)\right] \\
& \leq\left(\frac{f(a)+f(b)}{2}\right)\left[I_{\alpha}^{a} g(b)+{ }^{b} I_{\alpha} g(a)\right]
\end{aligned}
$$


$\alpha \in(n, n+1]$.

Proof. Since $f$ is a convex function on $[a, b]$, we have for all $t \in[0,1]$

$$
\begin{aligned}
2 f\left(\frac{a+b}{2}\right) & =2 f\left(\frac{t a+(1-t) b+t b+(1-t) a}{2}\right) \\
& \leq \frac{f(t a+(1-t) b)+f(t b+(1-t) b)}{2}
\end{aligned}
$$

Multiplying both sides of (2.2) by $2 t^{\alpha}(1-t)^{\alpha-n-1} g(t b+(1-t) a)$ then integrating the resulting inequality with respect to $t$ over $[0,1]$, we obtain

$$
\begin{aligned}
& 2 f\left(\frac{a+b}{2}\right) \int_{0}^{1} t^{n}(1-t)^{\alpha-n-1} g(t b+(1-t) a) d t \\
& \leq \int_{0}^{1} t^{n}(1-t)^{\alpha-n-1}[f(t a+(1-t) b)+f(t b+(1-t) b)] g(t b+(1-t) a) d t \\
& =\int_{0}^{1} t^{n}(1-t)^{\alpha-n-1} f(t a+(1-t) b) g(t b+(1-t) a) d t \\
& \quad+\int_{0}^{1} t^{n}(1-t)^{\alpha-n-1} f(t b+(1-t) a) g(t b+(1-t) a) d t
\end{aligned}
$$

Setting $x=t b+(1-t) a$, we get

$$
\begin{aligned}
& \frac{2}{(b-a)^{\alpha}} f\left(\frac{a+b}{2}\right) \int_{a}^{b}(x-a)^{n}(b-x)^{\alpha-n-1} g(x) d x \\
& \leq \frac{1}{(b-a)^{\alpha}}\left\{\int_{a}^{b}(x-a)^{n}(b-x)^{\alpha-n-1} f(a+b-x) g(x) d x\right. \\
& \left.\quad+\int_{a}^{b}(x-a)^{n}(b-x)^{\alpha-n-1} f(x) g(x) d x\right\} \\
& =\frac{1}{(b-a)^{\alpha}}\left\{\int_{a}^{b}(b-x)^{n}(x-a)^{\alpha-n-1} f(x) g(a+b-x) d x\right. \\
& \left.\quad+\int_{a}^{b}(x-a)^{n}(b-x)^{\alpha-n-1} f(x) g(x) d x\right\} \\
& =\frac{1}{(b-a)^{\alpha}}\left\{\int_{a}^{b}(b-x)^{n}(x-a)^{\alpha-n-1} f(x) g(x) d x\right. \\
& \left.\quad+\int_{a}^{b}(x-a)^{n}(b-x)^{\alpha-n-1} f(x) g(x) d x\right\}
\end{aligned}
$$


Therefore, by Lemma 1 we have

$$
\frac{n !}{(b-a)^{\alpha}} f\left(\frac{a+b}{2}\right)\left[I_{\alpha}^{a} g(b)+{ }^{b} I_{\alpha} g(a)\right] \leq \frac{n !}{(b-a)^{\alpha}}\left[I_{\alpha}^{a}(f g)(b)+{ }^{b} I_{\alpha}(f g)(a)\right]
$$

and the first inequality is proved. For the proof of the second inequality in (2.1) we first note that if $f$ is a convex function, then, for all $t \in[0,1]$, it yields

$$
f(t a+(1-t) b)+f(t b+(1-t) a) \leq f(a)+f(b) .
$$

Then multiplying both sides of (3.3) by $2 t^{\alpha}(1-t)^{\alpha-n-1} g(t b+(1-t) a)$ and integrating the resulting inequality with respect to $t$ over $[0,1]$, we obtain

$$
\begin{aligned}
& \int_{0}^{1} t^{n}(1-t)^{\alpha-n-1} f(t a+(1-t) b) g(t b+(1-t) a) d t \\
& \quad+\int_{0}^{1} t^{n}(1-t)^{\alpha-n-1} f(t b+(1-t) a) g(t b+(1-t) a) d t \\
& \leq[f(a)+f(b)] \int_{0}^{1} t^{n}(1-t)^{\alpha-n-1} g(t b+(1-t) a) d t
\end{aligned}
$$

i.e.

$\frac{n !}{(b-a)^{\alpha}}\left[I_{\alpha}^{a}(f g)(b)+{ }^{b} I_{\alpha}(f g)(a)\right] \leq \frac{n !}{(b-a)^{\alpha}}\left(\frac{f(a)+f(b)}{2}\right)\left[I_{\alpha}^{a} g(b)+{ }^{b} I_{\alpha} g(a)\right]$

The proof is completed.

Remark 1. If we take $\alpha=n+1$ in Theorem 2.1, then we obtain Theorem 2.2 in [2].

\section{Hermite-HAdAmARD-Fejér TYPE INEQUALITIES FOR CONFORMABLE} FRACTIONAL INEQUALITIES

Lemma 2. Let $f:[a, b] \rightarrow \mathbb{R}$ be a differentiable mapping on $(a, b)$ with $a<b$ and $f^{\prime} \in L[a, b]$. If $g:[a, b] \rightarrow \mathbb{R}$ is integrable and symmetric to $(a+b) / 2$ then the following equality holds for conformable fractional integral

$$
\begin{aligned}
& \left(\frac{f(a)+f(b)}{2}\right)\left[I_{\alpha}^{a} g(b)+{ }^{b} I_{\alpha} g(a)\right]-\left[I_{\alpha}^{a}(f g)(b)+{ }^{b} I_{\alpha}(f g)(a)\right] \\
& =\frac{(b-a)^{\alpha+1}}{n !} \int_{0}^{1} k(t) f^{\prime}((1-t) a+t b) d t
\end{aligned}
$$

where

$$
k(t)=\left[\int_{0}^{t} s^{n}(1-s)^{\alpha-n-1} g((1-s) a+s b) d s+\int_{1}^{t}(1-s)^{n} s^{\alpha-n-1} g((1-s) a+s b) d s\right]
$$

with $\alpha \in(n, n+1]$ 
Proof. It suffices to note that

$$
\begin{aligned}
I= & \int_{0}^{1} k(t) f^{\prime}(t) d t \\
= & \int_{0}^{1}\left[\int_{0}^{t} s^{n}(1-s)^{\alpha-n-1} g((1-s) a+s b) d s\right. \\
& \left.+\int_{1}^{t}(1-s)^{n} s^{\alpha-n-1} g((1-s) a+s b) d s\right] f^{\prime}((1-t) a+t b) d t \\
= & \int_{0}^{1}\left(\int_{0}^{t} s^{n}(1-s)^{\alpha-n-1} g((1-s) a+s b) d s\right) f^{\prime}((1-t) a+t b) d t \\
& +\int_{0}^{1}\left(\int_{1}^{t}(1-s)^{n} s^{\alpha-n-1} g((1-s) a+s b) d s\right) f^{\prime}((1-t) a+t b) d t \\
= & I_{1}+I_{2} .
\end{aligned}
$$

By integrating by parts and Lemma 1 we get

$$
\begin{aligned}
I_{1}= & \left.\left(\int_{0}^{t} s^{n}(1-s)^{\alpha-n-1} g((1-s) a+s b) d s\right) \frac{f((1-t) a+t b)}{b-a}\right|_{0} ^{1} \\
& -\int_{0}^{1} t^{n}(1-t)^{\alpha-n-1} g((1-t) a+t b) f((1-t) a+t b) d t \\
= & \left(\int_{0}^{1} s^{n}(1-s)^{\alpha-n-1} g((1-s) a+s b) d s\right) \frac{f(b)}{b-a} \\
& -\int_{0}^{1} t^{n}(1-t)^{\alpha-n-1}(f g)((1-t) a+t b) d t \\
= & \left(\int_{a}^{b}(x-a)^{n}(b-x)^{\alpha-n-1} g(x) d x\right) \frac{f(b)}{(b-a)^{\alpha+1}} \\
& -\frac{1}{(b-a)^{\alpha+1}} \int_{a}^{b}(x-a)^{n}(b-x)^{\alpha-n-1}(f g)(x) d x \\
= & \frac{n !}{(b-a)^{\alpha+1}}\left[I_{\alpha}^{a} g(b)+{ }^{b} I_{\alpha} g(a)\right] \frac{f(b)}{2}-\frac{n !}{(b-a)^{\alpha+1}}{ }^{b} I_{\alpha}(f g)(a)
\end{aligned}
$$

and similarly

$$
\begin{aligned}
I_{2}= & \left.\left(\int_{1}^{t}(1-s)^{n} s^{\alpha-n-1} g((1-s) a+s b) d s\right) \frac{f((1-t) a+t b)}{b-a}\right|_{0} ^{1} \\
& -\int_{0}^{1}(1-t)^{n} t^{\alpha-n-1} g((1-t) a+t b) f((1-t) a+t b) d t \\
= & \left(\int_{0}^{1}(1-s)^{n} s^{\alpha-n-1} g((1-s) a+s b) d s\right) \frac{f(a)}{b-a}
\end{aligned}
$$




$$
\begin{aligned}
& -\int_{0}^{1}(1-t)^{n} t^{\alpha-n-1}(f g)((1-t) a+t b) d t \\
= & \left(\int_{a}^{b}(b-x)^{n}(x-a)^{\alpha-n-1} g(x) d x\right) \frac{f(a)}{(b-a)^{\alpha+1}} \\
& -\frac{1}{(b-a)^{\alpha+1}} \int_{a}^{b}(b-x)^{n}(x-a)^{\alpha-n-1}(f g)(x) d x \\
= & \frac{n !}{(b-a)^{\alpha+1}}\left[I_{\alpha}^{a} g(b)+{ }^{b} I_{\alpha} g(a)\right] \frac{f(a)}{2}-\frac{n !}{(b-a)^{\alpha+1}} I_{\alpha}^{a}(f g)(b) .
\end{aligned}
$$

Thus, we can write

$$
\begin{aligned}
I= & I_{1}+I_{2} \\
= & \frac{n !}{(b-a)^{\alpha+1}} \\
& \times\left\{\left(\frac{f(a)+f(b)}{2}\right)\left[I_{\alpha}^{a} g(b)+{ }^{b} I_{\alpha} g(a)\right]-\left[I_{\alpha}^{a}(f g)(b)+{ }^{b} I_{\alpha}(f g)(a)\right]\right\} .
\end{aligned}
$$

Multiplying both sides by $\frac{(b-a)^{\alpha+1}}{n !}$ we obtain (3.1) which completes the proof.

Remark 2. If we take $\alpha=n+1$ in Lemma 2, then we obtain Lemma 2.4 in [2].

Theorem 2. Let $f: I \subseteq \mathbb{R} \rightarrow \mathbb{R}$ be a differentiable mapping on $I^{o}$ and $f^{\prime} \in L[a, b]$ with $a<b$. If $\left|f^{\prime}\right|$ is convex on $[a, b]$ and $g:[a, b] \rightarrow \mathbb{R}$ is continuous and symmetric to $(a+b) / 2$, then the following inequality for conformable fractional integrals holds

$$
\begin{aligned}
& \left|\left(\frac{f(a)+f(b)}{2}\right)\left[I_{\alpha}^{a} g(b)+{ }^{b} I_{\alpha} g(a)\right]-\left[I_{\alpha}^{a}(f g)(b)+{ }^{b} I_{\alpha}(f g)(a)\right]\right| \\
& \leq \frac{(b-a)^{\alpha+1}\|g\|_{\infty}}{n !}\left(\left|f^{\prime}(a)\right|+\left|f^{\prime}(b)\right|\right) \\
& \quad \times\left[\frac{1}{2} B(n+1, \alpha-n)+B_{1 / 2}(\alpha-n+1, n+1)+B_{1 / 2}(n+2, \alpha-n)\right]
\end{aligned}
$$

with $\alpha \in(n, n+1]$.

Proof. From Lemma 2 and the convexity of $\left|f^{\prime}\right|$, we have

$$
\begin{aligned}
& \left|\left(\frac{f(a)+f(b)}{2}\right)\left[I_{\alpha}^{a} g(b)+{ }^{b} I_{\alpha} g(a)\right]-\left[I_{\alpha}^{a}(f g)(b)+{ }^{b} I_{\alpha}(f g)(a)\right]\right| \\
& \leq \frac{(b-a)^{\alpha+1}}{n !} \int_{0}^{1}|k(t)|\left|f^{\prime}((1-t) a+t b)\right| d t \\
& \leq \frac{(b-a)^{\alpha+1}}{n !} \int_{0}^{1}|k(t)|\left[(1-t)\left|f^{\prime}(a)\right|+t\left|f^{\prime}(b)\right|\right] d t .
\end{aligned}
$$


Since $g:[a, b] \rightarrow \mathbb{R}$ is symmetric to $(a+b) / 2$ we write

$$
\begin{aligned}
& \int_{1}^{t}(1-s)^{n} s^{\alpha-n-1} g((1-s) a+s b) d s \\
& =\int_{0}^{1-t} s^{n}(1-s)^{\alpha-n-1} g(s a+(1-s) b) d s \\
& =\int_{0}^{1-t} s^{n}(1-s)^{\alpha-n-1} g((1-s) a+s b) d s
\end{aligned}
$$

then we have

$$
\begin{aligned}
|k(t)|= & \mid \int_{0}^{t} s^{n}(1-s)^{\alpha-n-1} g((1-s) a+s b) d s \\
& +\int_{1}^{t}(1-s)^{n} s^{\alpha-n-1} g((1-s) a+s b) d s \mid \\
= & \left|\int_{1-t}^{t} s^{n}(1-s)^{\alpha-n-1} g((1-s) a+s b) d s\right|
\end{aligned}
$$

so we get

$$
|k(t)| \leq\left\{\begin{array}{ll}
\int_{t}^{1-t} s^{n}(1-s)^{\alpha-n-1} d s, & t \in\left[0, \frac{1}{2}\right] \\
\int_{1-t}^{t} s^{n}(1-s)^{\alpha-n-1} d s, & t \in\left[\frac{1}{2}, 1\right]
\end{array} .\right.
$$

Combining (3.3) and (3.4), we get

$$
\begin{aligned}
& \left|\left(\frac{f(a)+f(b)}{2}\right)\left[I_{\alpha}^{a} g(b)+{ }^{b} I_{\alpha} g(a)\right]-\left[I_{\alpha}^{a}(f g)(b)+{ }^{b} I_{\alpha}(f g)(a)\right]\right| \\
& \leq \\
& \quad \times\left((1-t)\left|f^{\prime}(a)\right|+t\left|f^{\prime}(b)\right|\right) d t \\
& \quad+\frac{(b-a)^{\alpha+1}}{n !} \int_{1 / 2}^{1}\left(\int_{1-t}^{t}\left|s^{n}(1-s)^{\alpha-n-1} g((1-s) a+s b)\right| d s\right) \\
& \quad \times\left((1-t)\left|f^{\prime}(a)\right|+t\left|f^{\prime}(b)\right|\right) d t \\
& \leq \\
& \quad+\frac{(b-a)^{\alpha+1}\|g\|_{\infty}\left|f^{\prime}(a)\right|}{n !}\left[\int_{0}^{1 / 2}\left(\int_{t}^{1-t} s^{n}(1-s)^{\alpha-n-1} d s\right)(1-t) d t\right. \\
& \left.\quad+\frac{(b-a)^{\alpha+1}\|g\|_{\infty}\left|f^{\prime}(b)\right|}{n !}\left[\int_{0}^{t} s^{n}(1-s)^{\alpha-n-1} d s\right)(1-t) d t\right]
\end{aligned}
$$


$\left.+\int_{1 / 2}^{1}\left(\int_{1-t}^{t} s^{n}(1-s)^{\alpha-n-1} d s\right) t d t\right]$.

Using integration by parts, from equality (1.3) and (1.4)

$$
\begin{aligned}
& \int_{0}^{1 / 2}\left(\int_{t}^{1-t} s^{n}(1-s)^{\alpha-n-1} d s\right)(1-t) d t \\
& =\left.\left(\int_{t}^{1-t} s^{n}(1-s)^{\alpha-n-1} d s\right)\left(t-\frac{t^{2}}{2}\right)\right|_{0} ^{1 / 2} \\
& -\int_{0}^{1 / 2}\left[-(1-t)^{n} t^{\alpha-n-1}-t^{n}(1-t)^{\alpha-n-1}\right]\left(t-\frac{t^{2}}{2}\right) d t \\
& =B_{1 / 2}(\alpha-n+1, n+1)+\frac{1}{2} B_{1 / 2}(\alpha-n+2, n+1) \\
& +B_{1 / 2}(n+2, \alpha-n)+\frac{1}{2} B_{1 / 2}(n+3, \alpha-n), \\
& \int_{0}^{1 / 2}\left(\int_{t}^{1-t} s^{n}(1-s)^{\alpha-n-1} d s\right) t d t \\
& =\left.\left(\int_{t}^{1-t} s^{n}(1-s)^{\alpha-n-1} d s\right) \frac{t^{2}}{2}\right|_{0} ^{1 / 2} \\
& -\int_{0}^{1 / 2}\left[-(1-t)^{n} t^{\alpha-n-1}-t^{n}(1-t)^{\alpha-n-1}\right] \frac{t^{2}}{2} d t \\
& =\frac{1}{2} B_{1 / 2}(\alpha-n+2, n+1)+\frac{1}{2} B_{1 / 2}(n+3, \alpha-n) \text {. } \\
& \int_{1 / 2}^{1}\left(\int_{1-t}^{t} s^{n}(1-s)^{\alpha-n-1} d s\right)(1-t) d t \\
& =\left.\left(\int_{1-t}^{t} s^{n}(1-s)^{\alpha-n-1} d s\right)\left(t-\frac{t^{2}}{2}\right)\right|_{1 / 2} ^{1} \\
& -\int_{1 / 2}^{1}\left[t^{n}(1-t)^{\alpha-n-1}+(1-t)^{n} t^{\alpha-n-1}\right]\left(t-\frac{t^{2}}{2}\right) d t \\
& =\left.\left(\int_{1-t}^{t} s^{n}(1-s)^{\alpha-n-1} d s\right)\left(t-\frac{t^{2}}{2}\right)\right|_{1 / 2} ^{1} \\
& -\int_{0}^{1 / 2}\left[t^{n}(1-t)^{\alpha-n-1}+(1-t)^{n} t^{\alpha-n-1}\right]\left(\frac{1}{2}-\frac{t^{2}}{2}\right) d t \\
& =B(n+1, \alpha-n)-\frac{1}{2} B(n+1, \alpha-n)-B_{1 / 2}(\alpha-n, n+1)
\end{aligned}
$$


484

$$
\begin{aligned}
& -\frac{1}{2} B_{1 / 2}(n+3, \alpha-n)-\frac{1}{2} B_{1 / 2}(\alpha-n+2, n+1) \\
= & \frac{1}{2} B(n+1, \alpha-n)-\frac{1}{2} B_{1 / 2}(n+3, \alpha-n)-\frac{1}{2} B_{1 / 2}(\alpha-n+2, n+1)
\end{aligned}
$$

and

$$
\begin{aligned}
& \int_{1 / 2}^{1}\left(\int_{1-t}^{t} s^{n}(1-s)^{\alpha-n-1} d s\right) t d t \\
&=\left.\left(\int_{1-t}^{t} s^{n}(1-s)^{\alpha-n-1} d s\right) \frac{t^{2}}{2}\right|_{1 / 2} ^{1} \\
& \quad-\int_{1 / 2}^{1}\left[t^{n}(1-t)^{\alpha-n-1}+(1-t)^{n} t^{\alpha-n-1}\right] \frac{t^{2}}{2} d t \\
&=\left.\left(\int_{1-t}^{t} s^{n}(1-s)^{\alpha-n-1} d s\right) \frac{t^{2}}{2}\right|_{1 / 2} ^{1} \\
&-\int_{0}^{1 / 2}\left[t^{n}(1-t)^{\alpha-n-1}+(1-t)^{n} t^{\alpha-n-1}\right] \frac{(1-t)^{2}}{2} d t \\
&=\left.\left(\int_{1-t}^{t} s^{n}(1-s)^{\alpha-n-1} d s\right) \frac{t^{2}}{2}\right|_{1 / 2} ^{1} \\
&-\int_{0}^{1 / 2}\left[t^{n}(1-t)^{\alpha-n-1}+(1-t)^{n} t^{\alpha-n-1}\right]\left(\frac{1}{2}-t+\frac{t^{2}}{2}\right) d t \\
&=B(n+1, \alpha-n)-\frac{1}{2} B_{1 / 2}(n+1, \alpha-n)-\frac{1}{2} B_{1 / 2}(\alpha-n, n+1) \\
&+B_{1 / 2}(\alpha-n+1, n+1)+B_{1 / 2}(n+2, \alpha-n) \\
&-\frac{1}{2} B_{1 / 2}(n+3, \alpha-n)-\frac{1}{2} B_{1 / 2}(\alpha-n+2, n+1) \\
&= \frac{1}{2} B(n+1, \alpha-n)+B_{1 / 2}(n+2, \alpha-n)+B_{1 / 2}(\alpha-n+1, n+1) \\
&-\frac{1}{2} B_{1 / 2}(n+3, \alpha-n)-\frac{1}{2} B_{1 / 2}(\alpha-n+2, n+1) . \\
& B(3.7),(3.8) a n d(3.9) i n(3), w g \\
&
\end{aligned}
$$

Using (3.6), (3.7), (3.8) and (3.9) in (3.5), we get

$$
\begin{aligned}
& \left|\left(\frac{f(a)+f(b)}{2}\right)\left[I_{\alpha}^{a} g(b)+{ }^{b} I_{\alpha} g(a)\right]-\left[I_{\alpha}^{a}(f g)(b)+{ }^{b} I_{\alpha}(f g)(a)\right]\right| \\
& \leq \frac{(b-a)^{\alpha+1}\|g\|_{\infty}\left|f^{\prime}(a)\right|}{n !}\left[B_{1 / 2}(\alpha-n+1, n+1)+\frac{1}{2} B_{1 / 2}(\alpha-n+2, n+1)\right. \\
& \quad+B_{1 / 2}(n+2, \alpha-n)+\frac{1}{2} B_{1 / 2}(n+3, \alpha-n)
\end{aligned}
$$




$$
\begin{aligned}
& \left.-\frac{1}{2} B_{1 / 2}(n+3, \alpha-n)-\frac{1}{2} B_{1 / 2}(\alpha-n+2, n+1)\right] \\
& +\frac{(b-a)^{\alpha+1}\|g\|_{\infty}\left|f^{\prime}(b)\right|}{n !}\left[B_{1 / 2}(\alpha-n+2, n+1)+B_{1 / 2}(n+3, \alpha-n)\right. \\
& -\frac{1}{2} B_{1 / 2}(n+3, \alpha-n)+B_{1 / 2}(n+2, \alpha-n)+B_{1 / 2}(\alpha-n+1, n+1) \\
& \left.-\frac{1}{2} B_{1 / 2}(n+3, \alpha-n)-\frac{1}{2} B_{1 / 2}(\alpha-n+2, n+1)\right] \\
= & \frac{(b-a)^{\alpha+1}\|g\|_{\infty}}{n !}\left(\left|f^{\prime}(a)\right|+\left|f^{\prime}(b)\right|\right) \\
& \times\left[\frac{1}{2} B(n+1, \alpha-n)+B_{1 / 2}(\alpha-n+1, n+1)+B_{1 / 2}(n+2, \alpha-n)\right.
\end{aligned}
$$

which completes the proof.

Remark 3. If we take $\alpha=n+1$ in Theorem 2, then we obtain Theorem 2.6 in [2].

Theorem 3. Let $f: I \subseteq \mathbb{R} \rightarrow \mathbb{R}$ be a differentiable mapping on $I^{o}$ and $f^{\prime} \in L[a, b]$ with $a<b$. If $\left|f^{\prime}\right|^{q}, q>1$, is convex on $[a, b]$ and $g:[a, b] \rightarrow \mathbb{R}$ is continuous and symmetric to $(a+b) / 2$, then the following inequality for fractional integrals holds

$$
\begin{aligned}
& \left|\left(\frac{f(a)+f(b)}{2}\right)\left[I_{\alpha}^{a} g(b)+{ }^{b} I_{\alpha} g(a)\right]-\left[I_{\alpha}^{a}(f g)(b)+{ }^{b} I_{\alpha}(f g)(a)\right]\right| \\
& \leq \frac{(b-a)^{\alpha+1}\|g\|_{\infty} 2^{1 / p}}{n !} \\
& \quad \times\left(\int_{0}^{1 / 2}\left[B_{t}(n+1, \alpha-n)^{p}-B_{1-t}(n+1, \alpha-n)^{p}\right] d t\right)^{1 / p} \\
& \quad \times\left(\frac{\left|f^{\prime}(a)\right|^{q}+\left|f^{\prime}(b)\right|^{q}}{2}\right)^{1 / q}
\end{aligned}
$$

for $\alpha \in(n, n+1]$, where $1 / p+1 / q=1$

Proof. Using Lemma 2, Holder's inequality, (3.4) and the convexity of $\left|f^{\prime}\right|^{q}$, it follows that

$$
\begin{aligned}
& \left|\left(\frac{f(a)+f(b)}{2}\right)\left[I_{\alpha}^{a} g(b)+{ }^{b} I_{\alpha} g(a)\right]-\left[I_{\alpha}^{a}(f g)(b)+{ }^{b} I_{\alpha}(f g)(a)\right]\right| \\
& \leq \frac{(b-a)^{\alpha+1}}{n !}\left(\int_{0}^{1 / 2}\left(\int_{t}^{1-t}\left|s^{n}(1-s)^{\alpha-n-1} g((1-s) a+s b)\right| d s\right)^{p} d t\right)^{1 / p} \\
& \quad \times\left(\int_{0}^{1 / 2}\left|f^{\prime}(1-t) a+t b\right|^{q} d t\right)^{1 / q}
\end{aligned}
$$




$$
\begin{aligned}
& +\frac{(b-a)^{\alpha+1}}{n !}\left(\int_{1 / 2}^{1}\left(\int_{1-t}^{t}\left|s^{n}(1-s)^{\alpha-n-1} g((1-s) a+s b)\right| d s\right)^{p} d t\right)^{1 / p} \\
& \times\left(\int_{1 / 2}^{1}\left|f^{\prime}(1-t) a+t b\right|^{q} d t\right)^{1 / q} \\
& \left.\leq \frac{(b-a)^{\alpha+1}\|g\|}{n !}\left(\int_{0}^{1 / 2}\left(\int_{t}^{1-t} s^{n}(1-s)^{\alpha-n-1}\right) d s\right)^{p} d t\right)^{1 / p} \\
& \times\left(\int_{0}^{1 / 2}\left[(1-t)\left|f^{\prime}(a)\right|^{q}+t\left|f^{\prime}(a)\right|^{q}\right] d t\right)^{1 / q} \\
& \left.+\frac{(b-a)^{\alpha+1}\|g\|}{n !}\left(\int_{0}^{1 / 2}\left(\int_{1-t}^{t} s^{n}(1-s)^{\alpha-n-1}\right) d s\right)^{p} d t\right)^{1 / p} \\
& \times\left(\int_{1 / 2}^{1}\left[(1-t)\left|f^{\prime}(a)\right|^{q}+t\left|f^{\prime}(a)\right|^{q}\right] d t\right)^{1 / q} \\
& =\frac{(b-a)^{\alpha+1}\|g\|\left[\left(\int_{0}^{1 / 2}\left[B_{1-t}(n+1, \alpha-n)^{p}-B_{t}(n+1, \alpha-n)^{p}\right] d t\right)^{1 / p}\right.}{n !}\left[\frac{\left|f^{\prime}(a)\right|^{q}+\left|f^{\prime}(b)\right|^{q}}{2}\right)^{1 / q} \cdot \\
& \left.+\left(\int_{1 / 2}^{1}\left[B_{t}(n+1, \alpha-n)^{p}-B_{1-t}(n+1, \alpha-n)^{p}\right] d t\right)^{1 / p}\right] \\
& \times\left(\frac{\left|f^{\prime}(a)\right|^{q}+\left|f^{\prime}(b)\right|^{q}}{2}\right)^{1 / q} \\
& =
\end{aligned}
$$

Here we use

$$
(A-B)^{q} \leq A^{q}-B^{q}
$$

for any $A \geq B \geq 0$ and $q \geq 1$. The proof is completed.

Remark 4. If we take $\alpha=n+1$ in Theorem 3, then we obtain Theorem 2.9 (i) in [2].

Theorem 4. Let $f: I \subseteq \mathbb{R} \rightarrow \mathbb{R}$ be a differentiable mapping on $I^{o}$ and $f^{\prime} \in L[a, b]$ with $a<b$. If $\left|f^{\prime}\right|^{q}, q>1$, is convex on $[a, b]$ and $g:[a, b] \rightarrow \mathbb{R}$ is continuous and 
symmetric to $(a+b) / 2$, then the following inequality for fractional integrals holds

$$
\begin{aligned}
& \left|\left(\frac{f(a)+f(b)}{2}\right)\left[I_{\alpha}^{a} g(b)+{ }^{b} I_{\alpha} g(a)\right]-\left[I_{\alpha}^{a}(f g)(b)+{ }^{b} I_{\alpha}(f g)(a)\right]\right| \\
& \leq \frac{2(b-a)^{\alpha+1}\|g\|_{\infty}}{n !}\left(\int_{0}^{1}\left(\left|B_{t}(n+1, \alpha-n)-B_{1-t}(n+1, \alpha-n)\right|\right) d t\right)^{1-1 / q} \\
& \quad \times\left(\left[\frac{1}{2} B(n+1, \alpha-n)+B_{1 / 2}(\alpha-n+1, n+1)+B_{1 / 2}(n+2, \alpha-n)\right]\right)^{1 / q} \\
& \quad \times\left(\frac{\left|f^{\prime}(a)\right|^{q}+\left|f^{\prime}(b)\right|^{q}}{2}\right)^{1 / q}
\end{aligned}
$$

for $\alpha \in(n, n+1]$, where $1 / p+1 / q=1$.

Proof. Using Lemma 2, Power-mean inequality, (3.4) and the convexity of $\left|f^{\prime}\right|^{q}$, it follows that

$$
\begin{aligned}
& \left|\left(\frac{f(a)+f(b)}{2}\right)\left[I_{\alpha}^{a} g(b)+{ }^{b} I_{\alpha} g(a)\right]-\left[I_{\alpha}^{a}(f g)(b)+{ }^{b} I_{\alpha}(f g)(a)\right]\right| \\
& \leq \frac{1}{n !}\left(\int_{0}^{1}\left(\left|\int_{1-t}^{t} s^{n}(1-s)^{\alpha-n-1} g((1-s) a+s b) d s\right|\right) d t\right)^{1-1 / q} \\
& \quad \times\left((b-a)^{\alpha+1} \int_{0}^{1}\left(\left|\int_{1-t}^{t} s^{n}(1-s)^{\alpha-n-1} g((1-s) a+s b) d s\right|\right)\right. \\
& \left.\quad \times\left|f^{\prime}((1-t) a+t b)\right|^{q} d t\right)^{1 / q} \\
& \leq \frac{(b-a)^{\alpha+1}\|g\|_{\infty}}{(b-a)^{1 / q} n !}\left(\int_{0}^{1}\left(\left|\int_{1-t}^{t} s^{n}(1-s)^{\alpha-n-1} d s\right|\right) d t\right)^{1-1 / q} \\
& \quad \times\left(\int_{0}^{1}\left(\left|\int_{1-t}^{t} s^{n}(1-s)^{\alpha-n-1} d s\right|\right)\left[(1-t)\left|f^{\prime}(a)\right|^{q}+t\left|f^{\prime}(b)\right|^{q} d t\right)^{1 / q} .\right.
\end{aligned}
$$

We can write

$$
\begin{aligned}
& \int_{0}^{1}\left(\left|\int_{1-t}^{t} s^{n}(1-s)^{\alpha-n-1} d s\right|\right) d t \\
& =\int_{0}^{1}\left(\left|\int_{0}^{t} s^{n}(1-s)^{\alpha-n-1} d s-\int_{0}^{1-t} s^{n}(1-s)^{\alpha-n-1} d s\right|\right) d t \\
& =\int_{0}^{1}\left(\left|B_{t}(n+1, \alpha-n)-B_{1-t}(n+1, \alpha-n)\right|\right) d t
\end{aligned}
$$


and similarly to the proof of Theorem 2

$$
\begin{aligned}
& \int_{0}^{1}\left(\left|\int_{1-t}^{t} s^{n}(1-s)^{\alpha-n-1} d s\right|\right)\left[(1-t)\left|f^{\prime}(a)\right|^{q}+t\left|f^{\prime}(b)\right|^{q} d t\right. \\
& \leq\left(\left|f^{\prime}(a)\right|^{q}+\left|f^{\prime}(b)\right|^{q}\right)\left[\frac{1}{2} B(n+1, \alpha-n)\right. \\
& \left.\quad+B_{1 / 2}(\alpha-n+1, n+1)+B_{1 / 2}(n+2, \alpha-n)\right]
\end{aligned}
$$

We write (3.13) and (3.14) in (3.11), which completes the proof.

Remark 5. If we take $\alpha=n+1$ in Theorem 4, then we obtain Theorem 2.8 in [2].

\section{REFERENCES}

[1] T. Abdeljawad, “On conformable fractional calculus," J. Comput. Appl. Math., vol. 279, pp. 57-66, 2015, doi: 10.1016/j.cam.2014.10.016.

[2] I. Iscan, "Hermite-hadamard-fejer type inequalities for convex functions via fractional integrals," Stud. Univ. Babes-Bolyai Math., vol. 60, no. 3, pp. 355-366, 2015.

[3] R. Khalil, M. A. Horani, A. Yousef, and M. Sababheh, "A new definition of fractional derivative," J. Comput. Appl. Math., vol. 264, pp. 65-70, 2014, doi: 10.1016/j.cam.2014.01.002.

[4] E. Rainville, Special Functions. The Mcmillan Company, New York, 1960.

Authors' addresses

Erhan Set

Ordu University, Department of Mathematics, Faculty of Arts and Sciences, 52200 Ordu, Turkey E-mail address: erhanset@yahoo.com

\section{Ilker Mumcu}

Ordu University, Department of Mathematics, Faculty of Arts and Sciences, 52200 Ordu, Turkey

E-mail address: mumcuilker@msn.com 